\title{
Tetrandrine attenuates hyperoxia-induced lung injury in newborn rats via NF-kB p65 and ERK1/2 pathway inhibition
}

\author{
Beibei Jiao, Yan Tang, Shan Liu, Chunyan Guo \\ Department of Pediatrics, The first Affiliated Hospital of Henan University of Science and Technology, Luoyang, China \\ Contributions: (I) Conception and design: C Guo; (II) Administrative support: C Guo; (III) Provision of study materials or patients: S Liu; (IV) \\ Collection and assembly of data: B Jiao; (V) Data analysis and interpretation: B Jiao, Y Tang; (VI) Manuscript writing: All authors; (VII) Final \\ approval of manuscript: All authors. \\ Correspondence to: Chunyan Guo. Department of Pediatrics, The First Affiliated Hospital of Henan University of Science and Technology, 636 \\ Guanlin Road, Luolong District, Luoyang, China. Email: guochunyanluoy@aliyun.com.
}

\begin{abstract}
Background: Bronchopulmonary dysplasia (BPD) is an important cause of respiratory illness in preterm newborns that results in significant morbidity and mortality. Hyperoxia is a critical factor in the pathogenesis of BPD, hyperoxia-induced lung injury model has similar pathological manifestations as human BPD. Tetrandrine (Tet) is known to suppress oxidative stress, apoptosis and inflammation. Thus it has been used to prevent organ injuries. However, the protective effect of Tet against hyperoxia-induced lung injury in newborn rats has not been reported.
\end{abstract}

Methods: A hyperoxia-induced lung injury model was established using newborn rats exposed to high $\mathrm{O}_{2}$ levels. The models were treated with various concentrations of Tet, and a lung function test was conducted. Then, the lung tissues and blood were collected to detect the effect of Tet on cell apoptosis, inflammatory response, and fibrosis. The effect of Tet on nuclear factor-kappa $\mathrm{B}(\mathrm{NF}-\kappa \mathrm{B})$ and extracellular signal-regulated kinase1/2 (ERK1/2) pathways was also determined.

Results: Lung function was decreased in hyperoxia-induced rats, and Tet could reverse this inhibiting effect. For oxidative stress, Tet caused an increase in the levels of antioxidant enzymes. The apoptosis rate and apoptosis-related proteins were decreased in hyperoxia-induced rats after Tet treatment. Additionally, Tet treatment could reduce inflammatory factor levels, while increasing $\mathrm{CD} 4^{+} \mathrm{IFN}-\gamma^{+} \mathrm{T}$ cell levels and decreasing $\mathrm{CD}^{+} \mathrm{IL}-4^{+} \mathrm{T}$ cell levels. Tet treatment was also able to inhibit the expression of fibrosis-related markers and NF- $\kappa \mathrm{B}$ and ERK1/2 pathways.

Conclusions: Tet demonstrated potent activity against hyperoxia-induced lung injury in newborn rats through NF- $\mathrm{KB}$ and ERK1/2 pathway inhibition.

Keywords: Tetrandrine; bronchopulmonary dysplasia (BPD); hyperoxia; lung injury; NF-кB; ERK1/2

Submitted Jul 05, 2020. Accepted for publication Aug 07, 2020.

doi: $10.21037 / \mathrm{atm}-20-5573$

View this article at: http://dx.doi.org/10.21037/atm-20-5573

\section{Introduction}

Bronchopulmonary dysplasia (BPD) is a developmental disorder of the lung which is characterized by enlarged alveoli, arrested lung growth, and abnormal lung function (1). BPD primarily occurs in infants born when their lungs are still transitioning from the canalicular to saccular stage. It is an important cause of respiratory illness in preterm newborns that results in significant morbidity and mortality (2). Several studies have shown that BPD not only decreases lung function during infancy but also has a considerable impact on lung function later in childhood and may increase the likelihood of developing asthma (2). Thus, preventing or ameliorating BPD is important for infants at this susceptible stage. However, treatments for BPD, such as glucocorticoids and diuretics, have limited clinical 
efficiency, and thus novel treatment strategies are urgently needed.

The pathogenesis of BPD is complicated, with hyperoxia-induced lung injury being a major contributor to its pathogenesis (3). Hyperoxia can cause lung dynamic changes, precipitate alveolar endothelial and epithelial cell death, and even increase alveolar vascular permeability (4). The pathological changes of lung injury caused by hyperoxia exposure mainly include cell apoptosis or necrosis, massive recruitment of inflammatory cells, and fiber deposition (4). In animal models, hyperoxia-exposed neonatal lungs were observed to exhibit a severely simplified and enlarged alveolar structure, resembling the pathological changes observed in BPD (4,5). Meanwhile, the present strategies for inhibiting hyperoxia-induced lung injury, including using anti-oxidants, growth factors, or stem cells, have been shown to prevent the development of $\mathrm{BPD}(6,7)$.

Tetrandrine (Tet) is a bisbenzylisoquinoline alkaloid extracted from the root of Stephania tetrandra and other related species of Menispermaceae, and is known to possess anti-inflammatory, antiallergic, and antihypertensive properties (8). As a clinical drug in China, Tet has been used for decades to treat patients with inflammatory pulmonary diseases, autoimmune disorders, cardiovascular diseases, and hypertension. In recent years, Tet has been revealed to have a broad range of pharmacological actions, including antioxidant effects (9), anti-apoptosis effects (10) and antitumor activity (11). In the study conducted by Lin et al., Tet effectively impaired ovalbumin-induced airway inflammatory and oxidative stress in a rat model of asthma (9). In Ma's study, Tet was demonstrated to suppress the inflammation and apoptosis in aged rats, thus ameliorating sevoflurane induced cognitive impairment (10). Furthermore, due to its role on oxidative stress, apoptosis, and inflammation, Tet has been used to prevent organ injuries. Indeed, the protective effects of Tet against acute cardiac injury, ischemia-reperfusion ( $\mathrm{I} / \mathrm{R})$ injury, and brain injury have been reported in previous studies (12-14).

In addition, it has been reported that Tet treatment can suppress nuclear factor-kappa B (NF- $\mathrm{kB})$ and extracellular signal-regulated kinase (ERK) signaling pathways $(15,16)$. $\mathrm{NF}-\kappa \mathrm{B}$ is a ubiquitous transcriptional factor participating in the regulation of different down-stream genes involved in apoptosis, inflammation, and cell survival, which can affect the pathogenesis of numerous respiratory diseases, including asthma, cystic fibrosis, and acute lung injury (17). The NF$\kappa B$ family consists of five subunits, namely, p65, c-Rel, RelB, $\mathrm{p} 50$, and p52. Among these, $\mathrm{p} 65$ is the main transcriptional factor (17). Inhibiting NF- $\mathrm{BB}$ signaling in newborn mice has been demonstrated to alleviate hyperoxia-induced lung injury (1). Meanwhile, ERK $1 / 2$ is a crucial member of the mitogen-activated protein kinase (MAPK) family, which has been reported to be associated with defense signaling against injury (18). ERK1/2 is involved in the regulation of cell cycle progression, proliferation, differentiation, senescence, and apoptosis (18). It has been reported that high oxygen levels can induce the phosphorylation of ERK1/2 (19), and inhibiting ERK1/2 pathway has been shown to contribute to lung injury treatment (19).

Considering Tet's effects on oxidative stress, apoptosis,

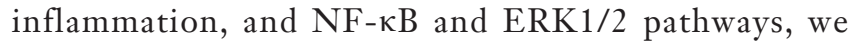
speculated that Tet might attenuate hyperoxia-induced lung injury, thus preventing the development of BPD. However, no study has yet reported whether Tet can influence the pathophysiology of hyperoxia-induced lung injury. We thus constructed hyperoxia-induced lung injury models in order to investigate the potential protective effect of Tet against hyperoxia-induced lung injury in newborn rats and attempt to elucidate its potential mechanism.

We present the following article in accordance with the ARRIVE reporting checklist (available at http://dx.doi. org/10.21037/atm-20-5573).

\section{Methods}

\section{Animal model and study groups}

Experiments were performed under a project license (No. SYXK2016-0006) granted by Institutional Animal Care and Use Committee of Henan University of Science and Technology, in compliance with the Guide for the Care and Use of Laboratory Animals (NIH Publication 86-23, National Academic Press, Washington, DC, USA, 1996). A total of 26 time-dated, pregnant Sprague-Dawley (SD) rats (230-300 g) were purchased from the experimental animal center of Henan University of Science and Technology (Henan, China). All animals were kept pathogen-free with water and food provided ad libitum, and were raised under conditions at $20-26^{\circ} \mathrm{C}, 40-70 \%$ relative humidity, 15 times/ $\mathrm{h}$ ventilation and a $12 \mathrm{~h}$ light/ $12 \mathrm{~h}$ dark cycle.

A total of 125 full-term newborn rats were randomly and equally assigned to the hyperoxia group $(n=100)$ and control group $(\mathrm{n}=25)$ within $12 \mathrm{~h}$ after birth. Then, rats in the hyperoxia group were further divided into four groups to receive different doses of Tet $(0,1.25,2.5$, and $5 \mathrm{mg} / \mathrm{kg}$; $\mathrm{n}=25$ each). Tet (C38H42N2O6, MW: 622.75, purity 
$\geq 98 \%$ ) was purchased from Selleck Chemicals (Houston, TX, USA). Rats in the hyperoxia group were placed in a closed oxygen tank with an atmosphere containing $85 \% \mathrm{O}_{2}$ for 14 days as described previously (20). Meanwhile, rats in the control group were maintained in room air $(21 \%$ oxygen). The half-life of Tet for oral administration in rats was $(20.6 \pm 3.7) \mathrm{h}(21)$. Thus, the rats in the Tet-treated groups were orally administered with Tet once a day for 14 days, while rats in the control and hyperoxia only groups were orally given an equal volume of $0.5 \%$ sodium carboxymethylcellulose solution for 14 days.

\section{Lung function test}

Fourteen days after treatment, minute ventilation, airway resistance and lung volume were tested as described previously (22). First, rats in each group were anesthetized with $10 \%$ pentobarbital sodium. Then, the rats were ventilated and placed into a body plethysmography box (Beijing Bestlab High-Tech Co., Ltd., Beijing, China). A Medlab biological signal acquisition and processing system recorder (Nanjing Medese Science and Technology Co., Ltd, Nanjing, China) was used to record the airway velocity, trans-pulmonary pressure, minute ventilation, and lung volume in anesthetized rats. Airway resistance was calculated as the ratio of transpulmonary pressure to respiratory airflow velocity.

After that, rats in each group were sacrificed by cervical dislocation. The right lobes of the lung were excised, washed with phosphate-buffered saline (PBS), blotted, and then weighed to obtain the wet weight; lung tissues were then dried in an oven for $48 \mathrm{~h}$ at a constant temperature of $65{ }^{\circ} \mathrm{C}$ and weighed to obtain the dry weight. The ratio of W/D (wet weight/dry weight)was calculated. Subsequently, the left lungs were resected and fixed in $4 \%$ (v/v) paraformaldehyde, embedded in paraffin, and stained with hematoxylin and eosin (HE) solution. The lung injury scoring parameters, including edema, neutrophil infiltration, and alveolar hemorrhage, were scored in five randomly selected fields of each section (23).

\section{Measurement of oxidative stress markers}

Superoxide dismutase (SOD), lactate dehydrogenase (LDH), malondialdehyde (MDA), and glutathione (GSH) detection kits were obtained from Nanjing Jiancheng Institute of Biotechnology (Nanjing, China). Lung tissues were prepared to make 1:10 homogenates and then centrifuged at $10,000 \times \mathrm{g}\left(4^{\circ} \mathrm{C}\right)$ for $15 \mathrm{~min}$ to obtain supernatants for the determination of the levels of SOD, LDH, MDA, and GSH.

\section{TdT-mediated dUTP nick end labeling (TUNEL) staining and Masson's staining}

The cell apoptosis in each group was detected by TdT-mediated dUTP nick end labeling (TUNEL) staining (Beyotime, Shanghai, China) according to the manufacturer's instructions. TUNEL-positive cell numbers were counted automatically using Image J software.

To assess alveolitis and fibrosis, lung slices in each group were stained with Masson's trichrome kits (Fuzhou Maixin Biotechnology Development Co., Ltd., Fuzhou, China) according to the manufacturer's instructions. The deposition of total collagen was semiquantitatively analyzed by Image J system software.

\section{Enzyme-linked immunosorbent assay (ELISA)}

The blood samples in each group were collected to determine the levels of inflammatory cytokines in serum by ELISA. After immediate centrifugation $(1,800 \times \mathrm{g})$ for $10 \mathrm{~min}$ at $4{ }^{\circ} \mathrm{C}$, the serum was separated. Then, serum interleukin (IL)-10, tumor necrosis factor (TNF) $\alpha$, and inducible nitric oxide synthase (iNOS) levels were measured by ELISA kits (Beyotime) according to manufacturer's instructions.

Lung tissues were homogenized, and protein was extracted from the tissues. The levels of IL-10, TNFa, and iNOS were measured by ELISA kits (Beyotime) according to the manufacturer's instructions.

\section{Flow cytometry}

The lung tissues were homogenized, and the cells were then separated. The cells were treated with phorbol 12-myristate 13-acetate (PMA) (Sigma-Aldrich, St. Louis, MO, USA), ionomycin (Sigma-Aldrich), and brefeldin A (BD, NJ, USA) to reach a final concentration of $10 \mathrm{ng} / \mathrm{mL}, 0.5 \mu \mathrm{g} / \mathrm{mL}$, and $1 \mu \mathrm{L} / \mathrm{mL}$. Then, cells were incubated with FITC-antiCD4 (BD) at room temperature in the dark for $30 \mathrm{~min}$, washed with cell staining buffer, mixed with $500 \mu \mathrm{L}$ fixation/permeabilization solution (BD), and incubated at room temperature in the dark for $45 \mathrm{~min}$. After that, cells were incubated with PE-anti-IL-4 (BD) and APC-antiIFN- $\gamma(\mathrm{BD})$ at room temperature in the dark for $45 \mathrm{~min}$ and resuspended with $300 \mu \mathrm{L}$ flow washing liquid (BD). 
Samples were measured by Canto II flow cytometer (BD).

\section{Western blot}

Lung lysate extracts were prepared, and the protein concentration was determined by the bicinchoninic acid (BCA) method (Beyotime). The extracted proteins were separated by SDS-PAGE gel (10\%) and transferred to a polyvinylidene fluoride (PVDF) membrane (Millipore, Billerica, MA, USA) followed by $1 \mathrm{~h}$ of blocking at room temperature (RT) in 7\% non-fat milk of PBS with $0.07 \%$ Tween-20. Next, blots were incubated with primary antibodies overnight at $4{ }^{\circ} \mathrm{C}$. Specific primary antibodies against caspase 3 (1:100, Wuhan Boster Biological Technology, Wuhan, China), caspase 9 (1:100, Wuhan Boster), B-cell lymphoma 2 [Bcl-2, 1:1,000, Cell Signaling Technology (CST), MA, USA], Bcl2-associated X protein (Bax, 1:1,000, CST), transforming growth factor (TGF) $\beta(1: 1,1000$, CST), fibronectin $(1: 2,000$, CST), $\alpha$-smooth muscle actin ( $\alpha$-SMA, 1:1,000, CST), nuclear factor- $\kappa \mathrm{B}$ (NF-кB) P65 (1:1,1000, CST), phospho-NF-кB P65 (p-NF$\kappa \mathrm{B}$ P56, 1:1,000, CST), IKB $\alpha$ (1:500, CST), phosphoIKB $\alpha$ (p-IKB $\alpha$, CST), ERK1/2 (1:1,000, Wuhan Boster), and phospho-ERK1/2 (p-ERK1/2, 1:1,000, Wuhan Boster) were incubated. Then, membranes were incubated with immunoglobulin $\mathrm{G}$ ( $\mathrm{IgG}$ )-horseradish peroxidase (HRP)conjugated secondary antibodies (1:2,000, Wuhan Boster) for $1 \mathrm{~h}$ at RT. The bound secondary antibodies on the PVDF membrane were reacted with enhanced chemiluminescence (ECL) detection reagents (Beyotime). $\beta$-actin $(1: 2,000$, Wuhan Boster) was used as an internal loading control.

\section{Statistical analyses}

Data analyses were performed using the SPSS statistics 23.0 software. Data are expressed in terms of mean \pm standard deviation (SD). Statistical comparisons among multiple groups were assessed with one way analysis of variance (ANOVA) followed by the Student-Newman-Keuls test for multiple comparisons. A $\mathrm{P}$ value $<0.05$ was considered statistically significant.

\section{Results}

\section{Tet improved lung function in newborn rats after} byperoxia exposure

Compared with the rats in the control group, the rats in the hyperoxia group had significantly higher lung injury scores and W/D values $(\mathrm{P}<0.05)$. However, Tet could reverse the effect caused by hyperoxia in a dose-dependent manner. Rats in the hyperoxia + Tet $2.5 \mathrm{mg} / \mathrm{kg}$ and hyperoxia + Tet $5 \mathrm{mg} / \mathrm{kg}$ groups exhibited significantly lower lung injury scores and W/D values than those in the hyperoxia group $(\mathrm{P}<0.05$, Figure $1 A, B)$. Among the other lung function indictors, minute ventilation, airway resistance, and lung volume were all reduced after hyperoxia exposure, but reversed by pretreatment with Tet in a dose-dependent manner (Figure 1C,D,E). Our results indicated that Tet administered at the dose of 2.5 and $5 \mathrm{mg} / \mathrm{kg}$ could significantly improve lung function of newborn rats under hyperoxia exposure.

\section{Tet inbibited cell apoptosis caused by byperoxia-induced lung injury}

HE staining showed significant alveolar septum thickening, perivascular space edema, and inflammatory cell infiltration in the newborn rats that developed obvious lung injury after hyperoxia exposure (Figure 2A). Tet pretreatment could reverse this pathological change.

TUNEL staining was used in this study to evaluate apoptosis. The representative images in each group are shown in Figure $2 \mathrm{~A}$. As expected, hyperoxia exposure could induce cell apoptosis, with a high apoptosis rate in hyperoxia group. Tet attenuated apoptosis significantly in a dose-dependent manner, as shown in Figure $2 B$. The apoptosis rate in the hyperoxia + Tet $2.5 \mathrm{mg} / \mathrm{kg}$ group or the hyperoxia + Tet $5 \mathrm{mg} / \mathrm{kg}$ group was much lower than that in the hyperoxia group $(\mathrm{P}<0.05)$.

In addition, Tet also affected the expression of apoptosisrelated proteins. As shown in Figure 2C,D, hyperoxia group had the highest Bax/Bcl-2, cleaved caspase-3/caspase-3, and cleaved caspase-9/caspase-9 ratios among the five groups. These three ratios in the hyperoxia + Tet $2.5 \mathrm{mg} / \mathrm{kg}$ group and hyperoxia + Tet $5 \mathrm{mg} / \mathrm{kg}$ group were much lower than those in the hyperoxia group $(\mathrm{P}<0.05)$, further indicating that Tet treatment had a role on inhibiting cell apoptosis caused by hyperoxia.

\section{Tet had a role on oxidative stress and lung fibrosis in newborn rats with lung injury}

SOD and GSH are important endogenous antioxidant enzymes, while MDA and LDH are regarded as biomarkers of oxidative stress. Compared with the control group, a 
A

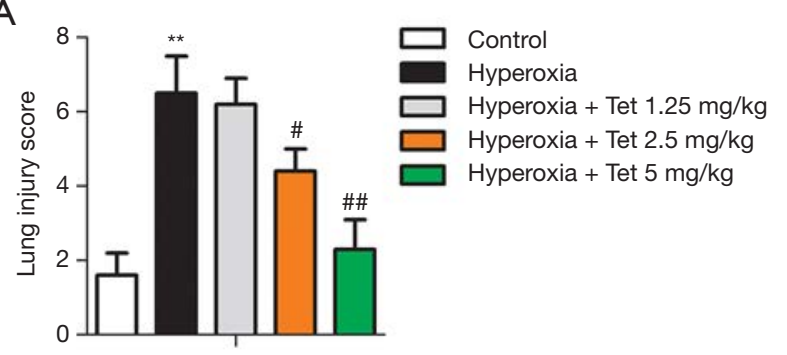

C

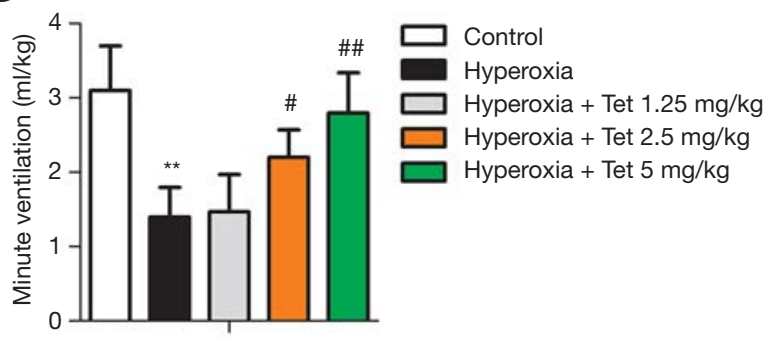

E

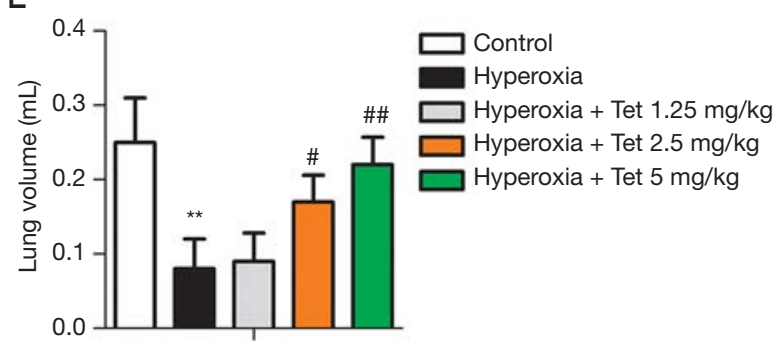

B

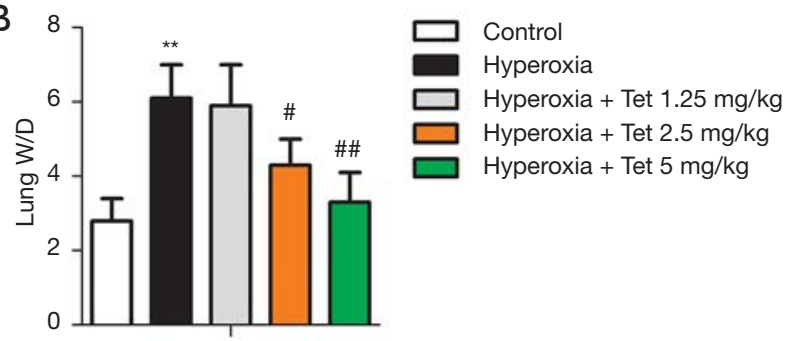

$\mathrm{D}$

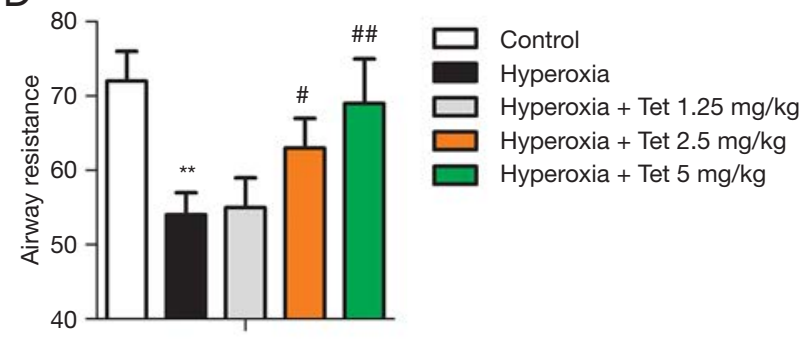

Figure 1 The effect of tetrandrine on lung function in newborn rats after hyperoxia exposure. The newborn rats were randomly and equally assigned to a hyperoxia group, hyperoxia + Tet $1.25 \mathrm{mg} / \mathrm{kg}$ group, a hyperoxia + Tet $2.5 \mathrm{mg} / \mathrm{kg}$ group, a hyperoxia + Tet $5 \mathrm{mg} / \mathrm{kg}$ group, and a control group ( $\mathrm{n}=25$, each) within $12 \mathrm{~h}$ after birth. (A) The lung injury scores measured in each group; (B) the W/D values measured in each group; (C) the minute ventilation value measured in each group; (D) the airway resistance value measured in each group; (E) the lung volume value measured in each group. ${ }^{* *}, \mathrm{P}<0.01$ vs. control group; ${ }^{\#}, \mathrm{P}<0.05$ vs. hyperoxia group; ${ }^{\# \#}, \mathrm{P}<0.01$ vs. hyperoxia group.

significant decrease in SOD and GSH levels and an increase in $\mathrm{LDH}$ and MDA levels were observed in the hyperoxia group $(\mathrm{P}<0.05)$, indicating that oxidative stress was present in hyperoxia-induced lung injury. Tet could increase the antioxidant enzymes levels and decrease the LDH and MDA levels in a dose-dependent manner (Figure $3 A, B, C, D$ ).

Masson staining revealed that the lung tissues in the control group showed no or very little thin filamentous fiber deposition, while lung tissues in the hyperoxia group showed a large amount of fiber deposition. However, the degree of fiber deposition in the three Tet pretreatment groups was significantly decreased in a dose-dependent manner (Figure 3E). Consistent with the pathological changes, the expressions of lung fibrosis markers (TGF- $\beta$, fibronectin, and $\alpha$-SMA) measured by Western blot were also reduced in a dose-dependent manner after Tet treatment (Figure 3F).

\section{Tet inbibited inflammation in newborn rats with lung injury}

The percentage of $\mathrm{CD} 4^{+} \mathrm{IFN}-\gamma^{+}$and $\mathrm{CD} 4^{+} \mathrm{IL}-4^{+} \mathrm{T}$ cells was determined by flow cytometry. As shown in Figure $4 A, B$, the number of $\mathrm{CD} 4^{+} \mathrm{IFN}-\gamma^{+} \mathrm{T}$ cells increased after hyperoxia exposure. However, Tet treatment could inhibit this trend. The number of $\mathrm{CD}^{+} \mathrm{IFN}-\gamma^{+} \mathrm{T}$ cells in the hyperoxia + Tet $2.5 \mathrm{mg} / \mathrm{kg}$ group and the hyperoxia + Tet $5 \mathrm{mg} / \mathrm{kg}$ group was much lower than that in the hyperoxia group $(\mathrm{P}<0.05)$. 


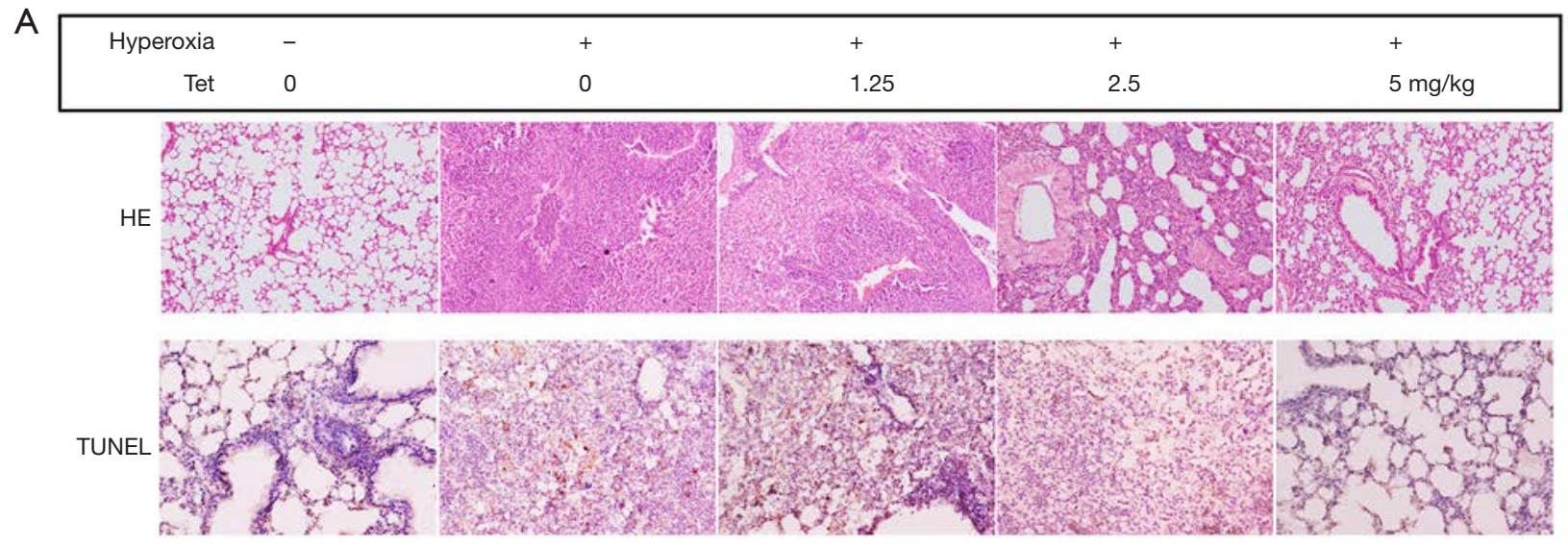

B

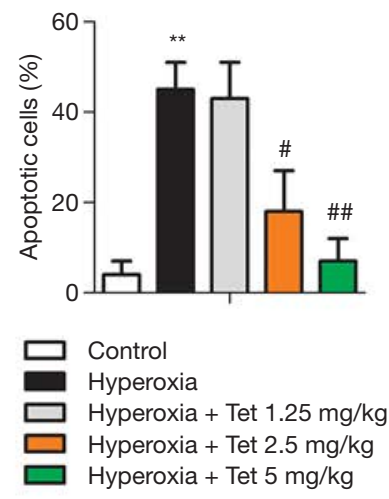

C

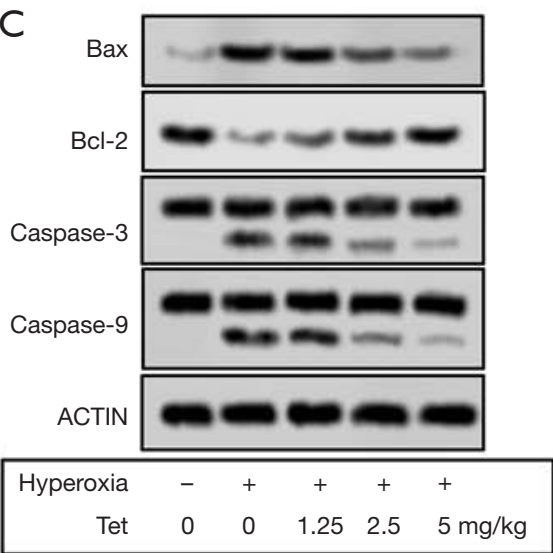

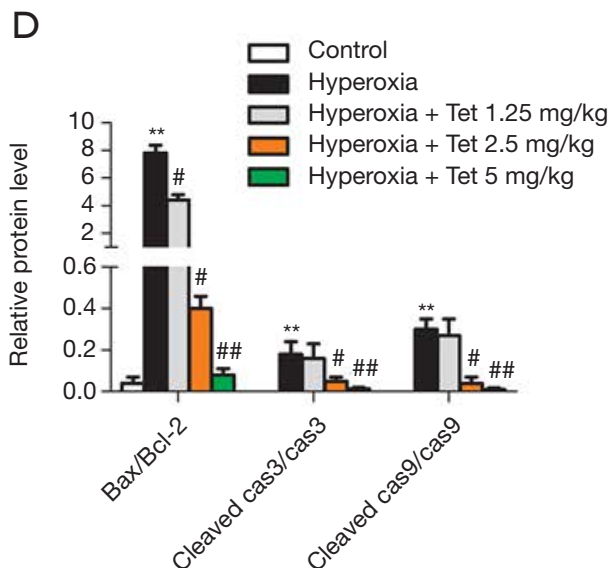

Figure 2 The effect of tetrandrine on cell apoptosis in newborn rats after hyperoxia exposure. (A) Representative images of HE staining and TUNEL staining ( $\times 400$ ); (B) the comparison of apoptosis rate in each group; (C) the expression of apoptosis-related proteins in each group measured by Western blot; (D) the comparison of apoptosis-related proteins in each group. ${ }^{* *}, \mathrm{P}<0.01$ vs. control group; ${ }^{*}, \mathrm{P}<0.05$ vs. hyperoxia group;,, $\mathrm{P}<0.01$ vs. hyperoxia group. TUNEL, terminal deoxynucleotidyl transferase (TdT)-mediated dUTP nick end labeling; Bcl-2, B-cell lymphoma 2; Bax, Bcl2-associated X protein.

Meanwhile, the number of CD4 $4^{+} \mathrm{LL}-4^{+} \mathrm{T}$ cells in each group showed the opposite trend (Figure 4C,D).

The levels of iNOS, TNF $\alpha$, and IL-10 in serum and lung tissues were determined by ELISA. Our results showed that the serum levels of iNOS and TNF $\alpha$ were increased after hyperoxia exposure. As expected, Tet could reduce the levels of these two inflammatory factors in a dose-dependent manner. Interestingly, the treatment of Tet at 2.5 and $5 \mathrm{mg} / \mathrm{kg}$ resulted in a significant increase of IL-10 levels (Figure 4E). Similarly, the levels of iNOS, TNF $\alpha$, and IL-10 in lung tissues in each group showed consistent results (Figure 4F).

\section{Tet inbibited NF-KB and ERK1/2 signaling pathway in newborn rats with lung injury}

As shown in Figure $5 A, B$, the expressions of p-NF- $\mathrm{kB}$ P65, $\mathrm{p}-\mathrm{IKB} \alpha$, and $\mathrm{p}$-ERK1/2 were increased after hyperoxia exposure. Tet treatment had a dose-dependent effect against hyperoxia exposure on the expressions of these three

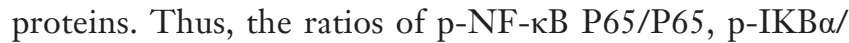
$\operatorname{IKB} \alpha$, and p-ERK1/2/ERK1/2 in the hyperoxia + Tet $2.5 \mathrm{mg} / \mathrm{kg}$ group and the hyperoxia + Tet $5 \mathrm{mg} / \mathrm{kg}$ group were much lower than those in the hyperoxia group $(\mathrm{P}<0.05$, Figure 5C,D,E). 
A

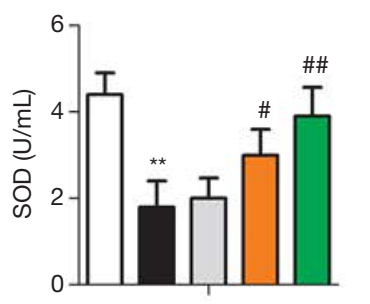

C

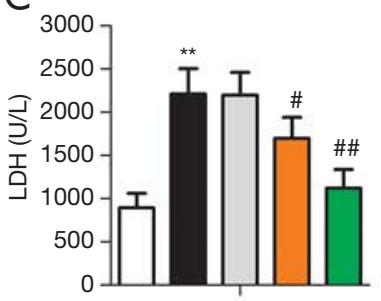

B

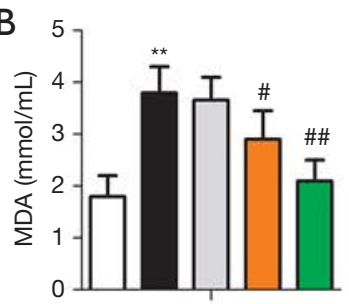

D

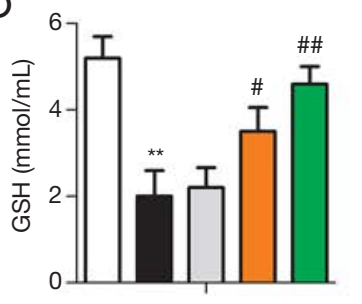

$\square$ Control

Hyperoxia

$\square$ Hyperoxia + Tet 1.25 mg/kg

$\square$ Hyperoxia + Tet $2.5 \mathrm{mg} / \mathrm{kg}$

$\square$ Hyperoxia + Tet $5 \mathrm{mg} / \mathrm{kg}$

E

$\square$ Control

$\square$ Hyperoxia + Tet $1.25 \mathrm{mg} / \mathrm{kg}$

$\square$ Hyperoxia + Tet $2.5 \mathrm{mg} / \mathrm{kg}$

$\square$ Hyperoxia + Tet $5 \mathrm{mg} / \mathrm{kg}$

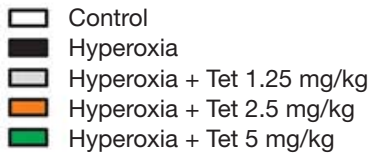

\begin{tabular}{|rlcccc|}
\hline Hyperoxia & - & + & + & + & + \\
Tet & 0 & 0 & 1.25 & 2.5 & $5 \mathrm{mg} / \mathrm{kg}$ \\
\hline
\end{tabular}

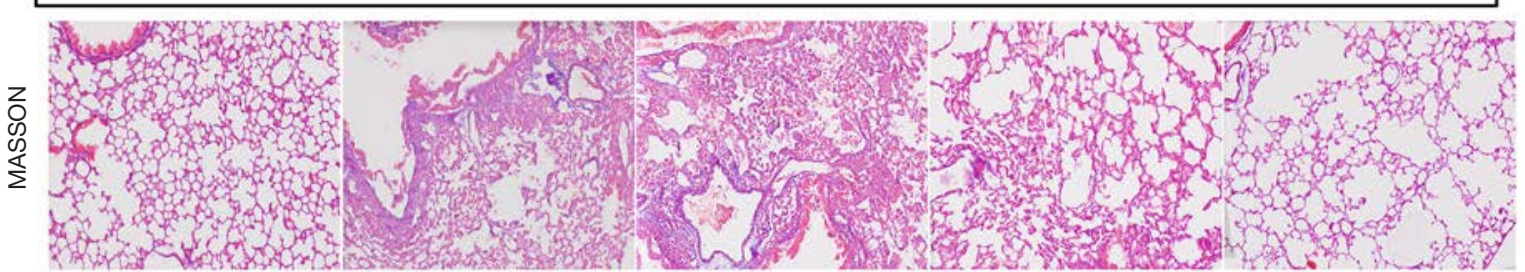

F
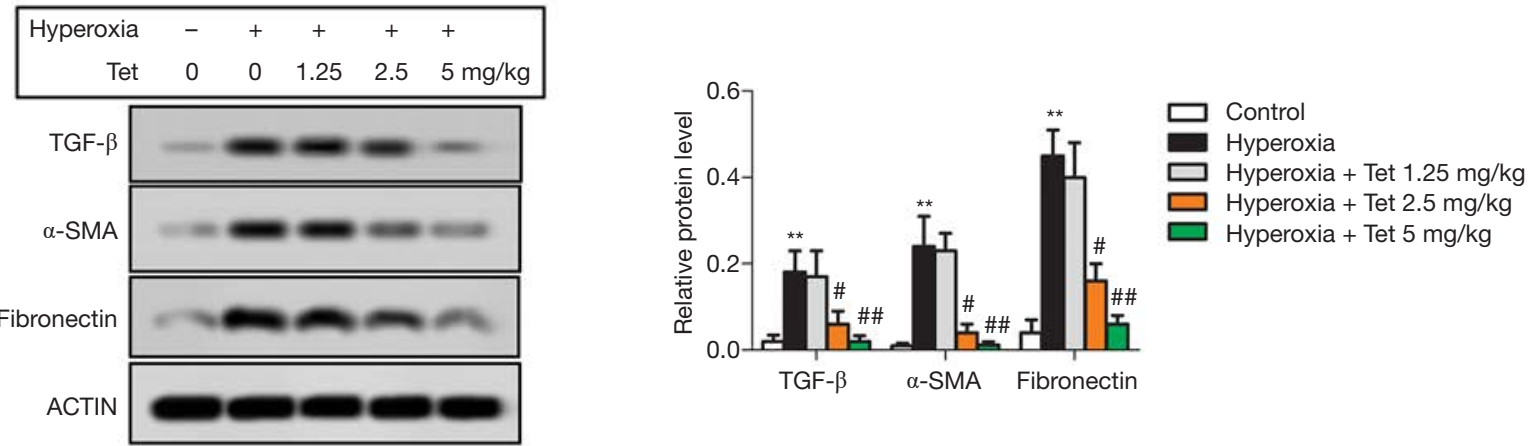

Figure 3 The effect of tetrandrine on oxidative stress and lung fibrosis in newborn rats with lung injury. (A) The expression levels of SOD in all groups; (B) the expression levels of MDA in all groups; (C) the expression levels of LDH in all groups; (D) the expression levels of GSH in all groups; (E) representative images of Masson staining in each group $(\times 400)$; (F) the expression of fibrosis-related proteins in each group measured by Western blot. **, $\mathrm{P}<0.01$ vs. control group; ${ }^{*}, \mathrm{P}<0.05$ vs. hyperoxia group; ${ }^{\#}, \mathrm{P}<0.01$ vs. hyperoxia group. SOD, superoxide dismutase; MDA, malondialdehyde; LDH, lactate dehydrogenase; GSH, glutathione; TGF- $\beta$, transforming growth factor $\beta$; $\alpha$-SMA, $\alpha$-smooth muscle actin. 
A

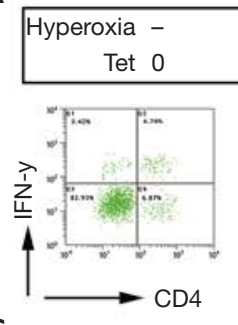

C

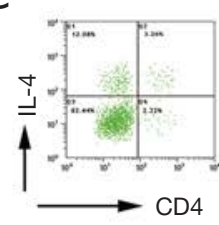

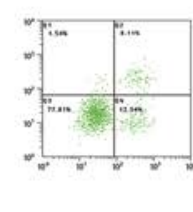

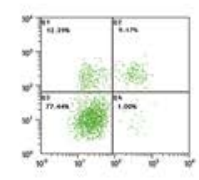

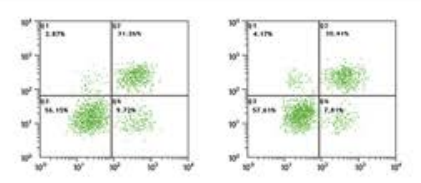
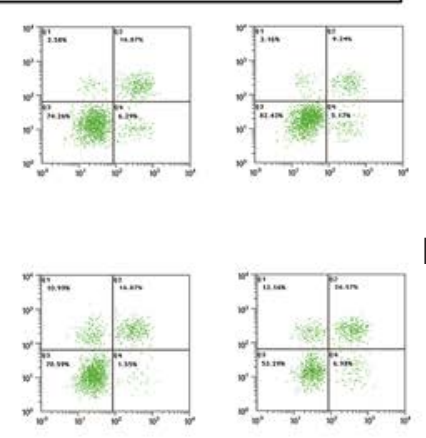

B
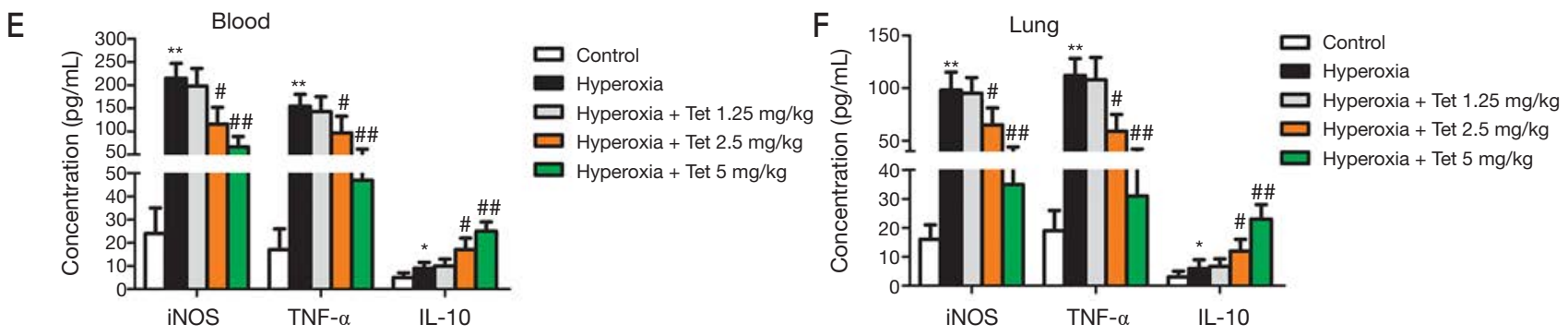

Figure 4 The effect of tetrandrine on inflammatory response in newborn rats with lung injury. (A) The percentage of $\mathrm{CD} 4^{+} \mathrm{IFN}-\gamma^{+} \mathrm{T}$ cells in each group measured by flow cytometry; (B) the comparison of $\mathrm{CD} 4^{+} \mathrm{IFN}-\gamma^{+} \mathrm{T}$ cells levels in each group; (C) the percentage of CD4 $4^{+} \mathrm{IL}-4^{+}$ $\mathrm{T}$ cells in each group measured by flow cytometry; (D) the comparison of CD4 $4^{+} \mathrm{IL}-4^{+} \mathrm{T}$ cells levels in each group; (E) the levels of iNOS, TNF $\alpha$, and IL-10 in serum in each group determined by ELISA; (F) the levels of iNOS, TNF $\alpha$, and IL-10 in lung tissues in each group determined by ELISA. *, $\mathrm{P}<0.05$ vs. control group; ${ }^{* *}, \mathrm{P}<0.01$ vs. control group; ${ }^{\prime}, \mathrm{P}<0.05$ vs. hyperoxia group; ${ }^{\# \prime}, \mathrm{P}<0.01$ vs. hyperoxia group. IL-10, interleukin 10; TNF $\alpha$, tumor necrosis factor $\alpha$; iNOS, inducible nitric oxide synthase.

\section{Discussion}

Hyperoxia-induced lung injury in newborn rats has long been used as a model of BPD $(5,24)$. Previous studies have observed lung function to be remarkably impaired in hyperoxia-induced BPD with an abnormal increase in airway resistance and a decrease in dynamic lung compliance $(25,26)$. In the present study, decreased lung function was also observed in newborn rats under hyperoxia exposure. However, Tet could reverse the impairment of lung function caused by hyperoxia in a dose-dependent manner.

Hyperoxia can increase reactive oxygen species (ROS) production and subsequently initiate oxidative stress. An increasing amount of evidence has indicated that oxidative stress plays a critical role in the initiation and progression of lung injury $(27,28)$. SOD and GSH are important endogenous antioxidant enzymes, which can remove the damaging ROS from the cellular environment (28). MDA and LDH are well-known oxidative products produced in cells. The imbalance between oxidative and antioxidative products can indirectly reflect the degree of oxidative stress (28). In our study, increased antioxidant enzymes levels and decreased antioxidative products levels after Tet pretreatment were observed, suggesting that Tet can reduce hyperoxia-induced oxidative stress.

Apart from oxidative stress, inflammation also plays an important role in the pathogenesis of hyperoxia-induced lung injury. Recently, studies have indicated that inhibiting the differentiation of proinflammatory $\mathrm{T}$ helper (CD4) cells may contribute to the anti-inflammatory effect of Tet (29). $\mathrm{CD}^{+}{ }^{+} \mathrm{T}$ cells induced by IFN- $\gamma$ differentiate into Th 1 cells to produce proinflammatory cytokines, while those induced by IL-4 differentiate into Th2 cells, secreting IL-4 and IL-10 to activate B cells (30). Additionally, Th1/ Th2 imbalance has been reported to play a role in the 
A

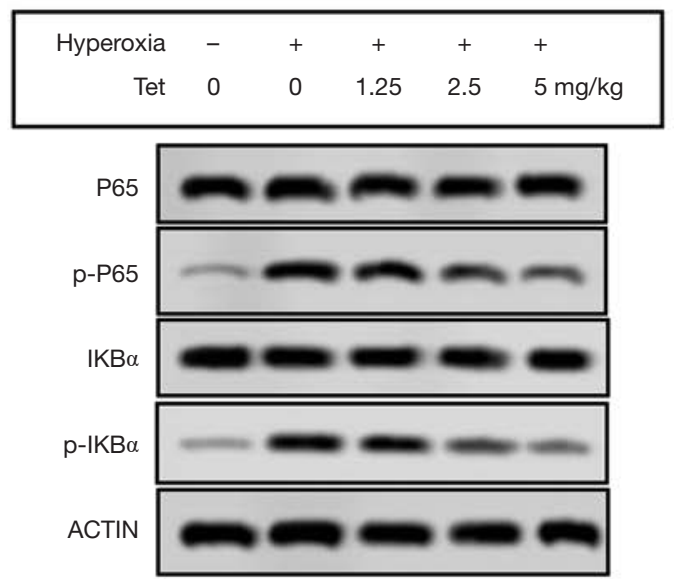

C
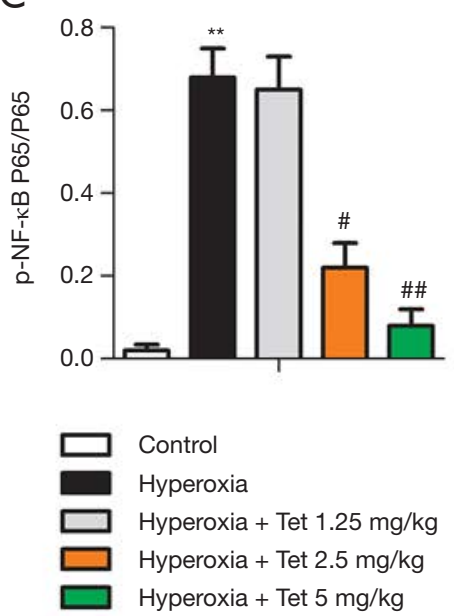

D

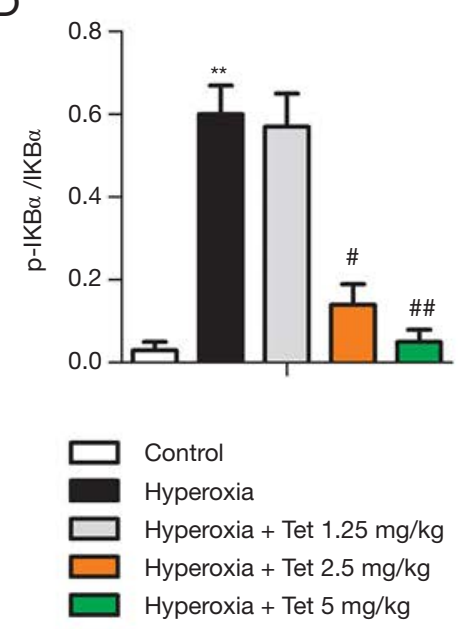

B

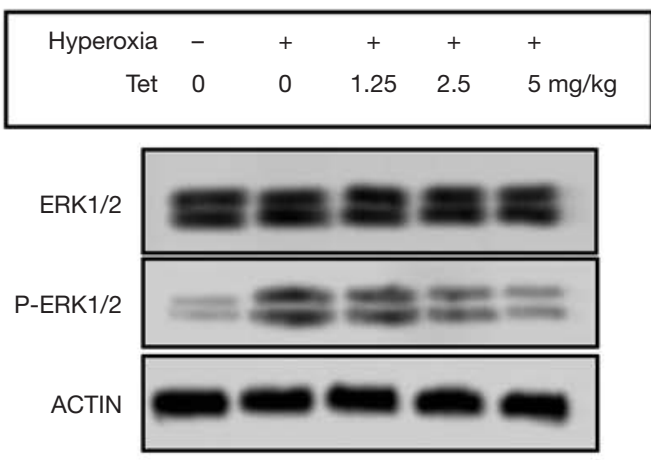

$\mathrm{E}$

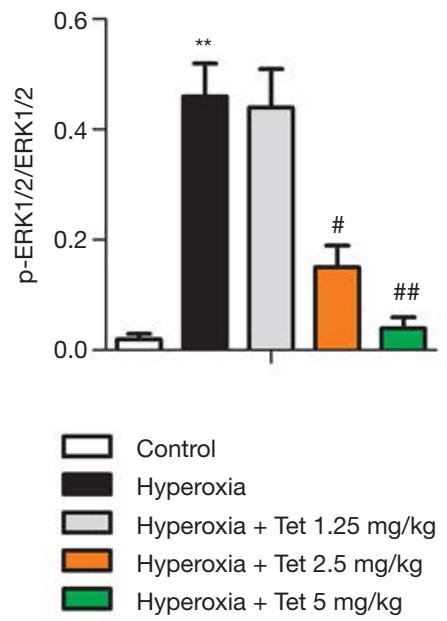

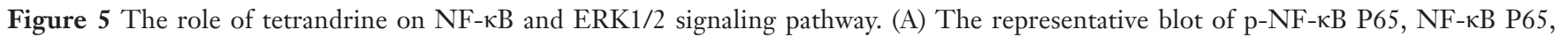
$\mathrm{p}-\mathrm{IKB} \alpha$, and IKB $\alpha$ expressions in each group; (B) the representative blot of $\mathrm{p}$-ERK1/2 and ERK1/2 expression in each group; (C) the ratio of p-NF-кB P65/P65 measured in each group; (D) the ratio of p-IKB $\alpha /$ IKB $\alpha$ measured in each group; (E) the ratio of p-ERK1/2/ERK1/2 measured in each group. ${ }^{* *}, \mathrm{P}<0.01$ vs. control group; ${ }^{\#}, \mathrm{P}<0.05$ vs. hyperoxia group; ${ }^{\# \#}, \mathrm{P}<0.01$ vs. hyperoxia group.

inflammation of lung injury (31). In this study, the increased $\mathrm{CD} 4^{+} \mathrm{IFN}-\gamma^{+} \mathrm{T}$ (Th1) cell level and decreased CD4 $4^{+} \mathrm{IL}-4^{+}$ T cells (Th2) level after Tet pretreatment were observed, indicating that Tet can regulate Th1/Th2 balance to inhibit inflammation. Furthermore, our study also found that Tet could affect the production of pro-inflammatory and anti-inflammatory cytokines. In mammals, iNOS and $\mathrm{TNF} \alpha$ are important pro-inflammatory factors produced by monocytes/macrophages, which can recruit and activate other immune cells and initiate inflammatory processes (32). In this study, the effect of Tet on reducing the levels of these two proinflammatory factors in hyperoxia-induced lung injury models was observed. Interestingly, we also found that Tet could increase IL-10 level. IL-10 is the one of the well-known anti-inflammatory cytokines which not only inhibit excessive cytokine release but also the control inflammatory process (32). The role of Tet in modulating proinflammatory and anti-inflammatory mediators further suggests that Tet can inhibit the inflammatory responses in a hyperoxia-induced lung injury model.

Hyperoxia is also one of the stimulatory factors of cell apoptosis. Apoptosis in lung epithelial cells has been regarded as an important pathological feature of hyperoxiainduced lung injury (33). As expected, a high apoptosis rate in rats under hyperoxia exposure was observed in the present study, and Tet significantly attenuated cell apoptosis in a dose-dependent manner. In addition, our results showed that Tet pretreatment could affect the expressions 
of apoptosis-related proteins. Bcl-2 and Bax both belong to the Bcl-2 family, performing opposing functions in the mitochondrial apoptotic pathway (34). Caspase-3 and caspase- 9 are members of the cysteine aspartic acidspecific protease (caspase) family. It has been proven that increased apoptosis is related to the enhanced expressions of cleaved caspase- 3 and cleaved caspase-9 (35). In this study, Bax, cleaved caspase- 3 , and cleaved caspase- 9 were downregulated after Tet pretreatment while Bcl-2 was upregulated, suggesting Tet may have a role in suppressing the apoptotic pathway.

Fibrosis is another classical pathological feature of lung injury. Inflammation, oxidative stress, and apoptosis are involved in the pathological process of lung fibrosis. A recent study has demonstrated that inhalation of Tet could alleviate inflammation and fibrosis in a bleomycininduced lung fibrosis rat model (36). In this study, the effect of Tet on alleviating fibrosis in newborn rats under hyperoxia exposure was also observed. The Masson staining revealed the degree of fiber deposition in Tet pretreatment groups to be significantly decreased in a dose-dependent manner (Figure 3E). Furthermore, the expressions of TGF- $\beta$, fibronectin, and $\alpha$-SMA were also reduced after Tet pretreatment. TGF- $\beta$, fibronectin, and $\alpha$-SMA are characteristic markers of pathological fibroblasts, which increase with the development of organ fibrosis (37). The inhibition of fibrosis markers of Tet indicates that Tet can inhibit the fibrosis formation pathway.

$\mathrm{NF}-\kappa \mathrm{B}$ is an inducible transcription factor that regulates the expression of many genes involved in immune, apoptosis, and inflammatory responses (38). The injury and inflammation of the lung tissues in hyperoxia-induced lung injury can be attenuated by inhibiting the activation of NF- $\kappa B$ (1). The activity of NF- $\kappa B$ is regulated primarily through interactions with inhibitory IKB proteins. At present, the best-studied NF- $\mathrm{kB}-\mathrm{IKB}$ interaction is that of $\mathrm{IKB} \alpha$ with the NF- $\mathrm{KB}$ p65 dimer. Activation of the NF$\kappa \mathrm{B}$ pathway triggers IKB kinase (IKK) activity, leading to phosphorylation and degradation of IKB proteins and the release of NF- $\mathrm{NB}$ p 65 . The released $\mathrm{p} 65 \mathrm{NF}-\mathrm{\kappa B}$ dimers translocate from the cytoplasm to the nucleus, binding to specific DNA sequences to promote transcription of target genes (39). Previous studies have reported that Tet could inhibit NF $\kappa \mathrm{B}$ pathway, thus suppressing inflammatory response $(15,40)$. For instance, Gao et al. found that Tet could block the NF- $\kappa$ B p65 in lipopolysaccharide (LPS)induced RAW 264.7 cells, inhibiting the expression of proinflammatory factors (40). In this study, we found that
Tet could decrease the ratios of $\mathrm{p}-\mathrm{NF}-\kappa \mathrm{B} \mathrm{P} 65 / \mathrm{P} 65$ and $\mathrm{p}-\mathrm{IKB} \alpha / \mathrm{IKB} \alpha$ in a dose-dependent manner, indicating that Tet can suppress this pathway. Furthermore, the effect of Tet on the inhibition of ERK1/2 was also observed. The ERK1/2 pathway is a signaling cascade that is involved in protecting against damage (41). It has been reported that hyperoxia significantly induces phosphorylation of ERK1/2 in lung tissue (19) and that the phosphorylation of ERK1/2 can mediate the secretion of proinflammatory chemokines and cytokines such as IL- 6 and TNF- $\alpha$ (19). Our results are consistent with other studies $(16,42)$ in that Tet could attenuate ERK1/2 phosphorylation. The effect of Tet on NF- $\kappa \mathrm{B}$ and ERK1/2 pathways is evidence that Tet can contribute to inhibiting the inflammatory response, apoptosis, oxidative stress, and fibrosis in hyperoxia-induced lung injury via these two pathways.

There are some limitations in this study. First, this study lacked a positive control group. Second, the optimal dose of Tet for hyperoxia-induced lung injury in newborn rats was not determined. Finally, because the cytotoxicity of Tet has been studied clearly in the previous studies (43), we did not investigate it in the present study. But the side effects of Tet in newborn rats should be determined in our further studies.

\section{Conclusions}

The results of our study demonstrated that Tet had potent activity against hyperoxia-induced lung injury in newborn rats. Tet significantly reduced oxidative stress, suppressed inflammatory responses, and inhibited cell apoptosis and fibrosis through inhibiting NF- $\mathrm{KB}$ and ERK1/2 signaling pathways. Our findings provide supporting evidence that Tet is a potential drug for preventing the development of $\mathrm{BPD}$, and thus warrants further investigation.

\section{Acknowledgments}

Funding: None.

\section{Footnote}

Reporting Checklist: The authors have completed the ARRIVE reporting checklist. Available at http://dx.doi. org/10.21037/atm-20-5573

Data Sharing Statement: Available at http://dx.doi. org/10.21037/atm-20-5573 
Conflicts of Interest: All authors have completed the ICMJE uniform disclosure form (available at http://dx.doi. org/10.21037/atm-20-5573). The authors have no conflicts of interest to declare.

Ethical Statement: The authors are accountable for all aspects of the work in ensuring that questions related to the accuracy or integrity of any part of the work are appropriately investigated and resolved. Experiments were performed under a project license (No. SYXK 2016-0006) granted by Institutional Animal Care and Use Committee of Henan University of Science and Technology, in compliance with the Guide for the Care and Use of Laboratory Animals (NIH Publication 86-23, National Academic Press, Washington, DC, USA, 1996).

Open Access Statement: This is an Open Access article distributed in accordance with the Creative Commons Attribution-NonCommercial-NoDerivs 4.0 International License (CC BY-NC-ND 4.0), which permits the noncommercial replication and distribution of the article with the strict proviso that no changes or edits are made and the original work is properly cited (including links to both the formal publication through the relevant DOI and the license). See: https://creativecommons.org/licenses/by-nc-nd/4.0/.

\section{References}

1. Li K, Zhang F, Wei L, et al. Recombinant Human Elafin Ameliorates Chronic Hyperoxia-Induced Lung Injury by Inhibiting Nuclear Factor-Kappa B Signaling in Neonatal Mice. J Interferon Cytokine Res 2020;40:320-30.

2. Hwang JS, Rehan VK. Recent Advances in Bronchopulmonary Dysplasia: Pathophysiology, Prevention, and Treatment. Lung 2018;196:129-38.

3. Maturu P, Wei-Liang Y, Androutsopoulos VP, et al. Quercetin attenuates the hyperoxic lung injury in neonatal mice: Implications for Bronchopulmonary dysplasia (BPD). Food Chem Toxicol 2018;114:23-33.

4. Wang J, Dong W. Oxidative stress and bronchopulmonary dysplasia. Gene 2018;678:177-83.

5. Tokuriki S, Igarashi A, Okuno T, et al. Treatment with Geranylgeranylacetone Induces Heat Shock Protein 70 and Attenuates Neonatal Hyperoxic Lung Injury in a Model of Bronchopulmonary Dysplasia. Lung 2017;195:469-76.

6. Amata E, Pittalà V, Marrazzo A, et al. Role of the Nrf2/
HO-1 axis in bronchopulmonary dysplasia and hyperoxic lung injuries. Clin Sci (Lond) 2017;131:1701-12.

7. Mo W, Li Y, Chang W, et al. The Role of LncRNA H19 in MAPK Signaling Pathway Implicated in the Progression of Bronchopulmonary Dysplasia. Cell Transplant 2020;29:963689720918294.

8. Bhagya N, Chandrashekar KR. Tetrandrine--A molecule of wide bioactivity. Phytochemistry 2016;125:5-13.

9. Lin $\mathrm{Y}, \mathrm{Yao} \mathrm{J}, \mathrm{Wu} \mathrm{M}$, et al. Tetrandrine Ameliorates Airway Remodeling of Chronic Asthma by Interfering TGF- $\beta 1 /$ Nrf-2/HO-1 Signaling Pathway-Mediated Oxidative Stress. Can Respir J 2019;2019:7930396.

10. Ma H, Yao L, Pang L, et al. Tetrandrine ameliorates sevoflurane-induced cognitive impairment via the suppression of inflammation and apoptosis in aged rats. Mol Med Rep 2016;13:4814-20.

11. Guo Y, Chen B, Pei X, et al. Radix Stephaniae Tetrandrine: An Emerging Role for Management of Breast Cancer. Curr Pharm Des 2020;26:25-36.

12. Li G, Li WR, Jin YG, et al. Tetrandrine Attenuated Doxorubicin-Induced Acute Cardiac Injury in Mice. Biomed Res Int 2020;2020:2616024.

13. Zhang TJ, Guo RX, Li X, et al. Tetrandrine cardioprotection in ischemia-reperfusion (I/R) injury via JAK3/STAT3/Hexokinase II. Eur J Pharmacol 2017;813:153-60.

14. Zhang L, Pang L, Zhu S, et al. Intranasal tetrandrine temperature-sensitive in situ hydrogels for the treatment of microwave-induced brain injury. Int J Pharm 2020;583:119384.

15. Bao G, Li C, Qi L, et al. Tetrandrine protects against oxygen-glucose-serum deprivation/reoxygenationinduced injury via PI3K/AKT/NF-kB signaling pathway in rat spinal cord astrocytes. Biomed Pharmacother 2016;84:925-30.

16. Qin G, Gui B, Xie J, et al. Tetrandrine Alleviates Nociception in a Rat Model of Migraine via Suppressing S100B and p-ERK Activation in Satellite Glial Cells of the Trigeminal Ganglia. J Mol Neurosci 2018;64:29-38.

17. Wu Y, Wang Y, Liu B, et al. SN50 attenuates alveolar hypercoagulation and fibrinolysis inhibition in acute respiratory distress syndrome mice through inhibiting NF- $\kappa \mathrm{B}$ p 65 translocation. Respir Res 2020;21:130.

18. Liu F, Yang X, Geng M, et al. Targeting ERK, an Achilles' Heel of the MAPK pathway, in cancer therapy. Acta Pharm Sin B 2018;8:552-62. 
19. Nie M, Wang Y, Lu Y, et al. Protective effects of fucoidan against hyperoxic lung injury via the ERK signaling pathway. Mol Med Rep 2018;17:1813-8.

20. Ruan Y, Dong W, Kang L, et al. The Changes of Twist1 Pathway in Pulmonary Microvascular Permeability in a Newborn Rat Model of Hyperoxia-Induced Acute Lung Injury. Front Pediatr 2020;8:190.

21. Song N, Zhang S, Li Q, et al. Establishment of a liquid chromatographic/mass spectrometry method for quantification of tetrandrine in rat plasma and its application to pharmacokinetic study. J Pharm Biomed Anal 2008;48:974-9.

22. Zhang Z, Cheng X, Ge D, et al. Protective Effects of Astragaloside IV Combined with Budesonide in Bronchitis in Rats by Regulation of Nrf2/Keap1 Pathway. Med Sci Monit 2018;24:8481-8.

23. Lin CH, Tsai CC, Chen TH, et al. Oxytocin maintains lung histological and functional integrity to confer protection in heat stroke. Sci Rep 2019;9:18390.

24. Özdemir ÖM, Taban Ö, Enli Y, et al. The effects of bosentan on hyperoxia-induced lung injury in neonatal rats. Pediatr Int 2019;61:1120-6.

25. Hansmann G, Fernandez-Gonzalez A, Aslam M, et al. Mesenchymal stem cell-mediated reversal of bronchopulmonary dysplasia and associated pulmonary hypertension. Pulm Circ 2012;2:170-81.

26. Sun Y, Chen C, Di T, et al. Human $\beta$-Defensin-2 Improves Hyperoxia-Induced Lung Structural and Functional Injury in Neonatal Rats. Med Sci Monit 2019;25:6074-84.

27. Cao F, Tian X, Li Z, et al. Suppression of NLRP3 Inflammasome by Erythropoietin via the EPOR/JAK2/ STAT3 Pathway Contributes to Attenuation of Acute Lung Injury in Mice. Front Pharmacol 2020;11:306.

28. Liu B, Chen A, Lan J, et al. Protective mechanism of 1-methylhydantoin against lung injury induced by paraquat poisoning. PLoS One 2019;14:e0222521.

29. Zou H, He T, Chen X. Tetrandrine inhibits differentiation of proinflammatory subsets of $T$ helper cells but spares de novo differentiation of iTreg cells. Int Immunopharmacol 2019;69:307-12.

30. Bing X, Xuelei L, Wanwei D, et al. EGCG Maintains Th1/ Th2 Balance and Mitigates Ulcerative Colitis Induced by Dextran Sulfate Sodium through TLR4/MyD88/NF-кB Signaling Pathway in Rats. Can J Gastroenterol Hepatol 2017;2017:3057268.

31. Song Q, Lin L, Chen L, et al. Co-administration of $\mathrm{N}$-acetylcysteine and dexmedetomidine plays a synergistic effect on protection of LPS-induced acute lung injury via correcting Th1/Th2/Th17 cytokines imbalance. Clin Exp Pharmacol Physiol 2020;47:294-301.

32. Wei H, Yin L, Feng S, et al. Dual-parallel inhibition of IL-10 and TGF- $\beta 1$ controls LPS-induced inflammatory response via NF- $\mathrm{NB}$ signaling in grass carp monocytes/ macrophages. Fish Shellfish Immunol 2015;44:445-52.

33. Bastug O, Fatih Sonmez M, Ozturk MA, et al. Effects of Lycopene in Hyperoxia-Induced Lung Injury in Newborn Rats. Int J Vitam Nutr Res 2018;88:270-80.

34. Abijeth B, Ezhilarasan D. Syringic acid induces apoptosis in human oral squamous carcinoma cells through mitochondrial pathway. J Oral Maxillofac Pathol 2020;24:40-5.

35. Yang Y, Zhang T, Zhou G, et al. Prevention of Necrotizing Enterocolitis through Milk Polar Lipids Reducing Intestinal Epithelial Apoptosis. J Agric Food Chem 2020;68:7014-23.

36. Su W, Liang Y, Meng Z, et al. Inhalation of Tetrandrinehydroxypropyl- $\beta$-cyclodextrin Inclusion Complexes for Pulmonary Fibrosis Treatment. Mol Pharm 2020;17:1596-607.

37. Mou F, Mou C. Necrostatin-1 Alleviates BleomycinInduced Pulmonary Fibrosis and Extracellular Matrix Expression in Interstitial Pulmonary Fibrosis. Med Sci Monit 2020;26:e919739.

38. Fang Y, Gao F, Liu Z. Angiotensin-converting enzyme 2 attenuates inflammatory response and oxidative stress

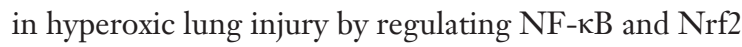
pathways. QJM 2019;112:914-24.

39. Simmons LJ, Surles-Zeigler MC, Li Y, et al. Regulation of inflammatory responses by neuregulin-1 in brain ischemia and microglial cells in vitro involves the NF-kappa B pathway. J Neuroinflammation 2016;13:237.

40. Gao LN, Feng QS, Zhang XF, et al. Tetrandrine suppresses articular inflammatory response by inhibiting pro-inflammatory factors via NF- $\kappa \mathrm{B}$ inactivation. J Orthop Res 2016;34:1557-68.

41. Mendell AL, MacLusky NJ. The testosterone metabolite $3 \alpha$-androstanediol inhibits oxidative stress-induced ERK phosphorylation and neurotoxicity in SH-SY5Y cells through an MKP3/DUSP6-dependent mechanism. Neurosci Lett 2019;696:60-6.

42. Ma JW, Zhang Y, Ye JC, et al. Tetrandrine Exerts a Radiosensitization Effect on Human Glioma through Inhibiting Proliferation by Attenuating ERK 
Phosphorylation. Biomol Ther (Seoul) 2017;25:186-93. 43. Alamzeb M, Omer M, Ur-Rashid M, et al. NMR, Novel Pharmacological and In Silico Docking Studies of Oxyacanthine and Tetrandrine: Bisbenzylisoquinoline
Alkaloids Isolated from Berberis glaucocarpa Roots. J Anal Methods Chem 2018;2018:7692913.

(English Language Editor: J. Gray)

Cite this article as: Jiao B, Tang Y, Liu S, Guo C. Tetrandrine attenuates hyperoxia-induced lung injury in newborn rats via NF- $\mathrm{kB}$ p 65 and ERK1/2 pathway inhibition. Ann Transl Med 2020;8(16):1018. doi: 10.21037/atm-20-5573 